\title{
Association of immunity markers with the risk of incident frailty: the Rugao longitudinal aging study
}

Hui Zhang ${ }^{1,2,3+}$, Meng Hao ${ }^{1,3+}$, Zixin Hü ${ }^{1,3,4+}$, Yi Li $i^{1,3}$, Xiaoyan Jiang ${ }^{5}$, Jiucun Wang ${ }^{1,3}$, Li Jin ${ }^{1,3}$, Zuyun Liu ${ }^{6}$, Xiaofeng Wang ${ }^{1,2^{*}}$ and Xuehui Sun ${ }^{1,2,3^{*}}$

\begin{abstract}
Background: The neutrophil-to-lymphocyte ratio (NLR), platelet-to-lymphocyte ratio (PLR) and systemic immuneinflammation index (SII) are readily available circulatory immunity markers that are associated with components of frailty. However, few studies have investigated the relationship between these immunity markers and frailty, and it remains unknown whether they are predictive of incident frailty in older adults in general. Hence, we aimed to examine the association of these immunity markers with the risk of incident frailty.

Results: Overall, 1822 older adults (mean age was $78.03 \pm 4.46$ years) were included in the Rugao Longitudinal Aging Study. NLR, PLR and SII were calculated from blood cell counts. The frailty definition was based on the Fried phenotype. At baseline, 200 (10.98\%) individuals were defined as frailty, and no significant associations of NLR, PLR and SII with frailty were found. During the 2-year follow-up, 180 (15.67\%) individuals were new-onset frailty. After adjustment, an increased logNLR (odds ratio [OR] 2.92, 95\% confidence interval [CI] 1.20-7.18), logPLR (OR 2.54, 95\% Cl: 1.01-6.53) and logSII (OR 2.34, 95\% Cl: 1.16-4.78) were significantly associated with a higher risk of incident frailty in all individuals. Additionally, the associations of $\operatorname{logNLR}(\mathrm{OR} 4.21$, 95\% Cl 1.54-11.62 logPLR (OR 3.38, 95\% Cl: 1.179.91) and logSII (OR 2.56, 95\% Cl: 1.15-5.72) with incident frailty were remained after excluding individuals with comorbidities. In further analyzed, individuals with higher levels of NLR and SII had higher risk of incident frailty when we stratified individuals by quartiles of these immunity markers.
\end{abstract}

Conclusion: NLR and SII are easily obtained immunity markers that could be used to predict incident frailty in clinical practice.

Keywords: Biomarkers, Epidemiology, Frailty, Immune function

\section{Background}

Frailty, a prominent phenotype of accelerated aging, is characterized by a loss of physiologic reserve and resistance to stressors due to cumulative declines in many physiological systems throughout the life course [1]. The prevalence of frailty is approximately $12 \%$ among

\footnotetext{
* Correspondence: wangxiaofeng@fudan.edu.cn; xhsun@fudan.edu.cn ${ }^{+}$Hui Zhang, Meng Hao and Zixin Hu contributed equally to this work. ${ }^{1}$ Human Phenome Institute, Fudan University, Shanghai, China

Full list of author information is available at the end of the article
}

individuals aged 50+ years [2]. Additionally, frailty is a significant risk factor for adverse outcomes, such as disability [3], multi-morbidity [4] and mortality [5]. It has become an emerging global health burden with major implications for clinical practice and public health [6]. While individuals with frailty are able to dynamically transition between states, it is important to identify the high-risk population with frailty and then prevent them from developing adverse outcomes.

(c) The Author(s). 2021 Open Access This article is licensed under a Creative Commons Attribution 4.0 International License, which permits use, sharing, adaptation, distribution and reproduction in any medium or format, as long as you give appropriate credit to the original author(s) and the source, provide a link to the Creative Commons licence, and indicate if changes were made. The images or other third party material in this article are included in the article's Creative Commons licence, unless indicated otherwise in a credit line to the material. If material is not included in the article's Creative Commons licence and your intended use is not permitted by statutory regulation or exceeds the permitted use, you will need to obtain permission directly from the copyright holder. To view a copy of this licence, visit http://creativecommons.org/licenses/by/4.0/ The Creative Commons Public Domain Dedication waiver (http://creativecommons.org/publicdomain/zero/1.0/) applies to the data made available in this article, unless otherwise stated in a credit line to the data. 
Age-associated changes in the immune system (characterized as a decline in immune function and an increase in low-grade, chronic systemic inflammation) have been suggested to be associated with frailty [7-9]. Neutrophils are important biomarkers of innate immunity, and platelets may contribute to immune function, whereas lymphocytes potentially reveal massive information about adaptive immunity [10]. A combination of these immune markers, including the neutrophil-tolymphocyte ratio (NLR), platelet-to-lymphocyte ratio (PLR) and systemic immune-inflammation index (SII), is thought to better reflect alterations in the immune system $[11,12]$. Evidence from observational studies has demonstrated a significant association of these circulatory markers of immunity with increased risks of dementia [13], cardiovascular disease [14] and mortality $[15,16]$ in older adults. Meanwhile, several studies have indicated a significant association of immune markers with frailty in specific populations, such as patients with coronary heart disease [17]. Additionally, associations of immune markers with precursor syndrome and/or components of frailty (including a slow gait speed and sarcopenia) have been reported [18-20]. However, in the general elderly population, few studies have investigated the association of these circulatory immunity markers with frailty, and it remains unknown whether they are predictive of incident frailty.

Therefore, to understand the association of these immunity markers (NLR, PLR and SII) with the risk of developing frailty, we first hypothesized that NLR, PLR and SII would be associated with frailty and then determined the longitudinal association of these immunity markers with the risk of incident frailty in older adults in general.

\section{Methods}

\section{Study population}

The Rugao Longitudinal Aging Study (RLAS) was an observational, prospective and community-based cohort study [21]. The first survey was conducted from November to December 2014 (wave 1). Then, the second survey was conducted in April 2016 (wave 2). The third and fourth surveys were conducted in November 2017 (wave




3) and December 2019 (wave 4), respectively. In the current study, the third wave was recognized as the baseline, and 1950 older adults were recruited. Among these participants, 120 individuals lacked frailty data, and 8 individuals lacked blood samples. In addition, 200 individuals were diagnosed as frailty. Hence, 1622 individuals were followed up during the subsequent 2 years. During the follow-up period, 67 participants died, and 406 participants lacked complete frailty information and/or withdrew from the study. Finally, 1149 participants were included and analyzed in this longitudinal setting (Fig. 1). The Human Ethics Committee of the School of Life Sciences of Fudan University, Shanghai, China, approved this study (No: BE1815). Written consent was obtained from all participants prior to the study.

\section{Outcomes}

\section{Fried's frailty phenotype}

The assessment of frailty status was conducted at baseline and at the 2-year follow-up. According to the frailty phenotype by Fried and colleagues, five criteria (including weight loss, weakness, exhaustion, slowness and low activity) were used to define frailty [22]. As previously reported [23], weight loss, exhaustion and low activity were measured by self-reported items. In detail, weight loss was defined if the participant responded "yes" to the question "Have you lost more than $4.5 \mathrm{~kg}$ or $5 \%$ of your body weight in the past 12 months?" Exhaustion was defined if the participant responded "yes" to the question "Have you felt tired at least 3 or 4 days per week?" Low activity was considered if the participant responded "yes" to the question: "Do you need help to walk?" Slowness was defined as being below the 20th percentile in the timed 'up and go' test (TUG). In the TUG, participants are asked to stand up from an armchair, walk $3 \mathrm{~m}$, return, and sit down again. The timing of the test begins when the participant's back is removed from the back of the chair and it stops when their buttocks touch the seat of the chair at the end of the test. Weakness was defined as being below the 20th specific percentile in maximum handgrip strength using a dynamometer (grip force, Shanghai Wanqing Electronics Co., Ltd. Shanghai, China) for three trials of each hand. The maximum value of the two hands was used in this study. Participants with any three or more indicators were defined as frail, one or two as 'prefrail' and none as'robust' [22].

\section{Measurements of immunity markers}

Blood samples were collected, and full blood count measurements were performed immediately after the blood draw. These measurements (including absolute counts of neutrophils, platelets and lymphocytes) were performed using an Olympus AU5811 clinical chemistry analyzer (Tokyo, Japan) with standard laboratory techniques. The neutrophil-to-lymphocyte ratio (NLR) was calculated based on the absolute neutrophil count $\left(\mathrm{N} ; \times 10^{9} / \mathrm{L}\right)$ and lymphocyte $\left(\mathrm{L} ; \times 10^{9} / \mathrm{L}\right)$ blood counts $(\mathrm{NLR}=\mathrm{N} / \mathrm{L})$. The platelet-to-lymphocyte ratio (PLR) was calculated for the absolute peripheral platelet $\left(\mathrm{P} ; \times 10^{9} / \mathrm{L}\right)$ and lymphocyte blood counts $(\mathrm{PLR}=\mathrm{P} / \mathrm{L})$. The systemic immuneinflammation index (SII) was calculated for the platelets and the NLR $(\mathrm{SII}=\mathrm{P} \times \mathrm{NLR})$ [11]. The NLR, PLR and SII were nonnormally distributed and were therefore log-transformed prior to performing the analyses.

\section{Covariates}

Demographic and clinical characteristics, laboratory data and lifestyles were collected. The demographic data included age, gender, marital status and educational status. Specifically, participants who married and lived together were categorized into the married group, while those who never married or were divorced, separated or widowed were assigned to the other group. Education status included illiteracy (never attended any school) and nonilliteracy. The clinical characteristics included selfreported cardiovascular disease (CVD), cancer and selfreported hypertension. Self-reported CVD included cerebral infarction, stroke, cerebral hemorrhage, coronary heart disease, myocardial infarction and heart failure. Comorbidities included self-reported CVD and cancer. Laboratory data included high-density lipoprotein (HDL), low-density lipoprotein (LDL), fasting blood glucose (FBG) and triglyceride (TG) levels. Lifestyles included smoking, alcohol consumption, regular exercise and body mass index (BMI). In brief, current and former smokers were assigned to the smoking group, while never smokers were categorized into no smoking groups; similarly, participants who self-reported current and former alcohol drinking were assigned to the drinking group, while those with never drinking were categorized into the no drinking group. Regular exercise was assessed by asking participants if their leisure time physical activity was more frequent than three times per week. The participants who responded "Yes" were recognized as engaging in regular exercise.

\section{Statistical analysis}

First, the characteristics of the study population were described at baseline. We also divided the NLR, PLR and SII into four groups according to the quartiles (Q). For NLR, Q1: $\leq 1.26 ; \mathrm{Q} 2:>1.26, \leq 1.71$; Q3: > 1.71, $\leq 2.35$; Q4: > 2.35 (Supplemental Table S1). For PLR, Q1: 71.07; Q2: > 71.07, $\leq 93.79$; Q3: > 93.79, $\leq 126.81$; Q4: > 126.81 (Supplemental Table S2). For SII: Q1: $\leq 213.61$; Q2: > 213.61, $\leq 309.45$; Q3: > 309.45, $\leq 445.76 ;$ Q4: > 445.76 (Supplemental Table S3). Then, we described the different characteristics of the study population between the quartiles of NLR, PLR and SII. Continuous and 
categorical variables are presented as the mean with standard deviation or frequency (\%), respectively. Group differences were analyzed by chi-square or ANOVA. Second, logistic regression models were conducted to evaluate the association of these inflammatory markers (NLR, PLR and SII) with frailty in all individuals. Third, to exclude the influences of comorbidities, these logistic regression models were reconstructed after excluding individuals with comorbidities. All analyses were conducted in two models. Model 1: unadjusted, Model 2: adjusted for age, gender, BMI, smoking, alcohol consumption, education, marital status, regular exercise, self-reported hypertension, triglyceride, HDL, LDL, FBG, frailty status at baseline (of longitudinal analyses) and comorbidity (of all individuals). A $p$ value (two-tailed) less than 0.05 was considered statistically significant. All analyses were conducted with SPSS 22.0 and R (version 3.6.1: www.r-project.org/).

\section{Results}

\section{Characteristics of the study population}

In our study, a total of 1822 (504 women) individuals were included and analyzed in cross-sectional setting, and their detailed information is shown in Table 1. Their mean age was $78.03 \pm 4.46$ years. Their mean NLR, PLR and SII were $1.97 \pm 1.12,104.26 \pm 50.56$ and $378.13 \pm$ 295.56, respectively. During the 2-year follow-up period, $180(15.67 \%)$ individuals were recognized as new-onset frailty.

\section{Cross-sectional association of the NLR, PLR and SII with frailty}

Supplemental Table S4 showed the cross-sectional association of the NLR, PLR and SII with frailty. There was significant association of $\operatorname{logNLR}$ (odds ratio [OR] 2.94, 95\% confidence interval [CI] 1.33-6.48) and $\operatorname{logSII}(\mathrm{OR}$ 2.12 , 95\% CI 1.13-3.98) with frailty were found in all individuals after adjusted for confounders. While, after excluded individuals with comorbidities, there were no significant association between these immunity marker and frailty (for logNLR: OR 2.35, 95\% CI 0.92-5.99; for logPLR: OR 1.82, 95\% CI 0.69-4.88; for logSII: OR 1.79, 95\% CI 0.85-3.76). Meanwhile, similarly results were found in further analyzed when we stratified individuals by quartiles of these immunity marker in with (Supplemental Table S5) and without comorbidities (Supplemental Table S 6).

\section{Longitudinal association between the NLR and incident frailty}

The associations of the NLR with incident frailty were explored in longitudinal settings. After controlling for confounding factors, there was significant association of increased $\operatorname{logNLR}(\mathrm{OR} 2.92,95 \% \mathrm{CI} 1.20-7.18, P$-value $=$
0.019) with incident frailty (Table 2). Individuals in higher NLR group (Q4) had higher risk (OR 2.39, 95\% CI $1.39-4.21, \quad P$-value $=0.002)$ of incident frailty (Table 3).

In addition, we further reanalyzed these associations between the NLR level and incident frailty among individuals without comorbidities (Table 4). With a 1-unit increase in the $\operatorname{logNLR}$, the OR for incident frailty was $4.21(95 \%$ CI 1.54-11.62, $P$-value $=0.005)$ after controlling for confounding factors (Table 2). Meanwhile, Individuals in higher NLR group (Q4) had higher risk (OR $2.88,95 \%$ CI $1.54-5.55, P$-value $=0.001)$ of incident frailty (Table 4).

\section{Longitudinal association between the PLR and incident frailty}

In crude models, with a 1-unit increase in the $\log P L R$, the risk for incident frailty was increased to 2.72 (95\% CI: 1.18-6.34, P-value =0.19) $($ Table 2). Similarly, consistent results were observed after adjusted for confounder factors in individuals with (OR 2.54, 95\% CI 1.01-6.53, $P$-value $=0.050)$ and without comorbidities (OR 3.38, 95\% CI 1.17-9.91, $P$-value $=0.025)$. In addition, we stratified individuals by quartiles of NLR in further analyzed. However, no significant associations were found in individuals with (Table 3 ) and without comorbidities (Table 4).

\section{Longitudinal association between the SII and incident frailty}

We first investigated the association of immune markers with frailty in all individuals. We found that the risk of incident frailty was $2.14(95 \% \mathrm{CI}$ : $1.14-4.03, P$-value $=0.018)$ with a 1-unit logSII increase in the crude model (Table 2). Individuals in the quartile 4 group had a higher risk of incident frailty $(\mathrm{OR}=1.70,95 \% \mathrm{CI}: 1.08-2.70, P$-value $=0.024)$ than those in the lowest SII group (Table 3). After adjustments, the association of SII with the risk of incident frailty remained in the quartile 4 groups (OR $=2.30,95 \% \mathrm{CI}: 1.32$ 4.08, $P$-value $=0.004$ ), and individuals with increased $\operatorname{logSII}$ had an increased risk of incident frailty $(\mathrm{OR}=2.34,95 \% \mathrm{CI}$ : $1.16-4.78$, P-value $=0.019$ ). Additionally, we further analyzed these associations of the SII with the risk of incident frailty among individuals without comorbidities (Tables 2 and 4). Similar results were found for the SII levels and incident frailty in the crude (per unit of logSII: OR $=2.50,95 \% \mathrm{CI}$ : 1.23-5.09, P-value $=0.012$ ) and adjusted models (per unit of $\operatorname{logSII}$ OR $=2.56,95 \%$ CI: $1.15-5.72$, P-value $=0.021$ ).

\section{Discussion}

\section{Principal findings}

In this longitudinal study, we investigated the predictive effect of circulatory markers of immunity for incident frailty in older adults. Our principal findings were that 
Table 1 Characteristics of the study population stratified by gender at baseline

\begin{tabular}{|c|c|c|c|c|c|}
\hline Characteristics & & Total $(N=1822)$ & Males $(n=848)$ & Females $(n=974)$ & $P$-value \\
\hline Age & $M \pm S D$, years & $78.03 \pm 4.46$ & $77.92 \pm 4.44$ & $78.13 \pm 4.47$ & 0.300 \\
\hline Body mass index & $\mathrm{M} \pm \mathrm{SD}, \mathrm{kg} / \mathrm{m}^{2}$ & $24.14 \pm 6.04$ & $23.86 \pm 3.31$ & $24.39 \pm 7.65$ & 0.062 \\
\hline \multirow[t]{2}{*}{ Smoking ${ }^{\neq}$} & Yes, n (\%) & $365(20.03 \%)$ & $327(38.56 \%)$ & $38(3.90 \%)$ & $<0.001$ \\
\hline & No, n (\%) & 1440 (79.03\%) & $510(63.80 \%)$ & 930 (95.48\%) & \\
\hline \multirow[t]{2}{*}{ Alcohol assumption ${ }^{\ddagger}$} & Yes, n (\%) & $553(29.25 \%)$ & 381 (44.93\%) & $172(17.66 \%)$ & $<0.001$ \\
\hline & No, n (\%) & $1246(68.39 \%)$ & $453(53.42 \%)$ & $793(81.41 \%)$ & \\
\hline \multirow[t]{2}{*}{ Educational status ${ }^{\neq}$} & Illiteracy & $879(48.24 \%)$ & $148(17.45 \%)$ & 731 (75.05\%) & $<0.001$ \\
\hline & Non-illiteracy & 942 (51.70\%) & 699 (82.43\%) & $243(24.95 \%)$ & \\
\hline \multirow[t]{2}{*}{ Marital status $s^{\&, \neq}$} & Married, n (\%) & $1171(64.27 \%)$ & $627(73.94 \%)$ & $544(55.85 \%)$ & $<0.001$ \\
\hline & Others ${ }^{\&}, \mathrm{n}(\%)$ & 607 (33.32\%) & $201(23.70 \%)$ & $406(41.68 \%)$ & \\
\hline \multirow[t]{2}{*}{ Regular exercise ${ }^{\ddagger}$} & Yes, n (\%) & $412(22.61 \%)$ & $238(28.07 \%)$ & $174(17.86 \%)$ & $<0.001$ \\
\hline & No, n (\%) & $1237(67.56 \%)$ & $531(62.62 \%)$ & 706 (72.48\%) & \\
\hline Triglyceride & $\mathrm{M} \pm \mathrm{SD}, \mathrm{mmol} / \mathrm{L}$ & $1.42 \pm 1.01$ & $1.22 \pm 0.88$ & $1.59 \pm 1.09$ & $<0.001$ \\
\hline High-density lipoprotein & $\mathrm{M} \pm \mathrm{SD}, \mathrm{mmol} / \mathrm{L}$ & $1.85 \pm 0.45$ & $1.84 \pm 0.45$ & $1.86 \pm 0.46$ & 0.371 \\
\hline Low-density lipoprotein & $\mathrm{M} \pm \mathrm{SD}, \mathrm{mmol} / \mathrm{L}$ & $2.80 \pm 0.63$ & $2.70 \pm 0.61$ & $2.88 \pm 0.64$ & $<0.001$ \\
\hline Fasting blood glucose & $\mathrm{M} \pm \mathrm{SD}, \mathrm{mmol} / \mathrm{L}$ & $5.83 \pm 1.50$ & $5.73 \pm 1.36$ & $5.92 \pm 1.60$ & 0.006 \\
\hline Neutrophil count & $\mathrm{M} \pm \mathrm{SD}, \times 10^{9} / \mathrm{L}$ & $3.53 \pm 1.36$ & $3.65 \pm 1.42$ & $3.43 \pm 1.29$ & 0.001 \\
\hline Lymphocytes count & $\mathrm{M} \pm \mathrm{SD}, \times 10^{9} / \mathrm{L}$ & $2.05 \pm 0.94$ & $2.00 \pm 1.11$ & $2.09 \pm 0.77$ & 0.036 \\
\hline Platelet count & $\mathrm{M} \pm \mathrm{SD}, \times 10^{9} / \mathrm{L}$ & $190.57 \pm 67.78$ & $182.11 \pm 61.25$ & $197.97 \pm 72.22$ & $<0.001$ \\
\hline NLR & $M \pm S D$ & $1.97 \pm 1.12$ & $2.12 \pm 1.27$ & $1.84 \pm 0.96$ & $<0.001$ \\
\hline PLR & $\mathrm{M} \pm \mathrm{SD}$ & $104.26 \pm 50.56$ & $103.01 \pm 50.05$ & $105.37 \pm 51.01$ & 0.319 \\
\hline SII & $\mathrm{M} \pm \mathrm{SD}$ & $378.13 \pm 295.56$ & $389.32 \pm 306.74$ & $368.56 \pm 285.39$ & 0.135 \\
\hline \multirow[t]{2}{*}{ Self-report hypertension } & Yes, n (\%) & 848 (46.54\%) & 371 (43.75\%) & 477 (48.97\%) & 0.015 \\
\hline & No, n (\%) & $974(53.46 \%)$ & 477 (56.25\%) & $497(51.03 \%)$ & 0.265 \\
\hline \multirow[t]{2}{*}{ Comorbidity $^{\mp}$} & Yes, n (\%) & 341 (18.72\%) & $153(18.04 \%)$ & $188(19.30 \%)$ & 0.269 \\
\hline & No, n (\%) & $1481(81.28 \%)$ & 695 (81.96\%) & 786 (80.70\%) & \\
\hline \multirow[t]{3}{*}{ Frailty status at baseline } & Robust, n (\%) & $583(32.00 \%)$ & $333(39.27 \%)$ & $250(25.66 \%)$ & $<0.001$ \\
\hline & Pre-Frailty, n (\%) & $1039(57.02 \%)$ & $452(53.30 \%)$ & $587(60.27 \%)$ & \\
\hline & Frailty, n (\%) & $200(10.98 \%)$ & $63(7.43 \%)$ & 137 (14.07\%) & \\
\hline
\end{tabular}

M: mean, SD: standard deviation. NLR: neutrophil-to-lymphocytes ratio, PLR: platelet-to-lymphocyte ratio, SII: systemic immune-inflammation index, Continuous and categorical variables were present as mean with SD and frequency (\%), Group difference were analyzed by chi-square or ANOVA test. \& including separated, divorced, never married or widowed. ${ }^{*}$ Unknown: Educational status (1, 0.05\%), Marital status (44, 2.41\%), Alcohol assumption (23, 1.26\%), Smoking: (17, 0.93\%), Regular exercise: $(173,9.50 \%) .{ }^{\mp}$ Including cerebral infarction, stroke, cerebral hemorrhage, coronary heart disease, myocardial infarction, heart failure and cancer

an elevated NLR and SII were robustly associated with an increased risk of incident frailty. These associations were even stronger in individuals without comorbidities. Additionally, because NLR and SII are extensively and readily obtainable in the laboratory and clinical fields, they may be proposed as predictors for the incidence of frailty.

\section{Compared with other studies}

To date, several studies have examined the association of immune markers with frailty by studying the NLR, PLR and SII in older adults in general. Collerton et al. conducted a cross-sectional study in the Newcastle 85+ Study and found inverse associations of memory/naïve CD8 $\mathrm{T}$ and $\mathrm{B}$ cell ratios with the frailty index and physical frailty [24]. In addition, several studies suggested a significant association of the NLR, PLR and SII levels with precursor syndrome and/or components of frailty. Gait speed is a feasible predictor of health-risk assessment in geriatrics [25-27] and is considered a hallmark of frailty. Previous studies reported a significant association between NLR levels and slow gait speed in older adults in general $(\mathrm{OR}=3.82,95 \% \mathrm{CI}: 1.87-7.89, P$ value $<0.001$ ) [18]. Additionally, similar significant associations were also found between the NLR level and a slow gait speed in patients with cancer (Spearman's correlation coefficient $[\mathrm{r}]:-0.48, P$ value $=0.0001)[28]$ and coronary heart disease $(\mathrm{r}: 0.211, P$-value $=0.001)$ [17], respectively. 
Table 2 Association of immunity markers with risk of incident frailty in non-frail individuals

\begin{tabular}{|c|c|c|c|c|}
\hline & \multicolumn{2}{|l|}{ Model 1} & \multicolumn{2}{|l|}{ Model 2} \\
\hline & OR $(95 \% \mathrm{Cl})$ & $P$-value & OR $(95 \% \mathrm{Cl})$ & $P$-value \\
\hline \multicolumn{5}{|l|}{ All individuals } \\
\hline NLR & $2.14(0.98,4.67)$ & 0.056 & $2.92(1.20,7.18)$ & 0.019 \\
\hline PLR & $2.72(1.18,6.34)$ & 0.019 & $2.54(1.01,6.53)$ & 0.050 \\
\hline SII & $2.14(1.14,4.03)$ & 0.018 & $2.34(1.16,4.78)$ & 0.019 \\
\hline Neutrophil count & $1.58(0.54,4.64)$ & 0.406 & $2.49(0.72,8.72)$ & 0.151 \\
\hline Platelet count & $2.39(0.81,7.36)$ & 0.122 & $1.70(0.51,6.00)$ & 0.398 \\
\hline Lymphocytes count & $0.34(0.12,0.99)$ & 0.048 & $0.30(0.09,1.00)$ & 0.051 \\
\hline \multicolumn{5}{|l|}{ Without comorbidities } \\
\hline NLR & $3.13(1.31,7.47)$ & 0.010 & $4.21(1.54,11.62)$ & 0.005 \\
\hline PLR & $3.58(1.39,9.30)$ & 0.009 & $3.38(1.17,9.91)$ & 0.025 \\
\hline SII & $2.50(1.23,5.09)$ & 0.012 & $2.56(1.15,5.72)$ & 0.021 \\
\hline Neutrophil count & $1.73(0.51,5.89)$ & 0.376 & $2.33(0.56,9.76)$ & 0.244 \\
\hline Platelet count & $2.03(0.60,7.19)$ & 0.261 & $1.32(0.33,5.47)$ & 0.697 \\
\hline Lymphocytes count & $0.18(0.06,0.59)$ & 0.005 & $0.14(0.04,0.54)$ & 0.004 \\
\hline
\end{tabular}

Sarcopenia is often considered a precursor syndrome or a physical component of frailty. Several studies have explored associations between these immunity markers and sarcopenia. Zhao et al. included 4224 middle-aged and older adults from the West China Health and Aging
Trend (WCHAT) study and examined these associations in a cross-sectional cohort. They found that a higher NLR $(\mathrm{OR}=1.123,95 \%$ CI: $1.047-1.205, P$-value $<0.01)$, PLR $(\mathrm{OR}=1.004,95 \%$ CI: $1.003-1.006$, P-value $<0.001)$ and SII $(\mathrm{OR}=1.001,95 \% \mathrm{CI}: 1.000-1.001, \mathrm{P}$-value <

Table 3 Association of immunity markers with risk of incident frailty in all individuals

\begin{tabular}{|c|c|c|c|c|c|}
\hline Immunity markers & Event/N (\%) & Model 1 & & Model 2 & \\
\hline & & OR $(95 \% \mathrm{Cl})$ & $P$-value & OR $(95 \% \mathrm{Cl})$ & $P$-value \\
\hline NLR & $180 / 1149$ (15.67\%) & & & & \\
\hline $\mathrm{Q} 1(\leq 1.26)$ & $41 / 292(14.04 \%)$ & Ref & Ref & Ref & Ref \\
\hline $\mathrm{Q} 2(>1.26, \leq 1.71)$ & $44 / 312(14.10 \%)$ & $1.00(0.63,1.59)$ & 0.995 & $1.16(0.67,2.03)$ & 0.590 \\
\hline Q3 $(>1.71, \leq 2.35)$ & $43 / 279(15.41 \%)$ & $1.12(0.70,1.78)$ & 0.644 & $1.33(0.77,2.33)$ & 0.309 \\
\hline $\mathrm{Q} 4$ (> 2.35) & $52 / 266(19.55 \%)$ & $1.49(0.95,2.34)$ & 0.082 & $2.39(1.39,4.21)$ & 0.002 \\
\hline PLR & $180 / 1149$ (15.67\%) & & & & \\
\hline $\mathrm{Q} 1(\leq 71.07)$ & $39 / 287$ (13.59\%) & Ref & Ref & Ref & Ref \\
\hline Q2 (> 71.07, $\leq 93.79)$ & $46 / 317(14.51 \%)$ & $1.08(0.68,1.72)$ & 0.745 & $0.98(0.57,1.71)$ & 0.954 \\
\hline Q3 $(>93.79, \leq 126.81)$ & 45/285 (15.79\%) & $1.19(0.75,1.89)$ & 0.468 & $1.08(0.63,1.84)$ & 0.787 \\
\hline Q4 (> 126.81) & $50 / 260(19.23 \%)$ & $1.51(0.96,2.40)$ & 0.075 & $1.35(0.79,2.32)$ & 0.273 \\
\hline SII & $180 / 1149$ (15.67\%) & & & & \\
\hline $\mathrm{Q} 1(\leq 213.61)$ & $38 / 295$ (12.88\%) & Ref & Ref & Ref & Ref \\
\hline Q2 (> 213.61, $\leq 309.45)$ & 45/307 (14.66\%) & $1.16(0.73,1.85)$ & 0.538 & $1.37(0.78,2.44)$ & 0.276 \\
\hline Q3 (> 309.45, $\leq 445.76)$ & 46/293 (15.70\%) & $1.23(0.77,1.97)$ & 0.380 & $1.55(0.89,2.73)$ & 0.126 \\
\hline Q4 (> 445.76) & $51 / 254(20.08 \%)$ & $1.70(1.08,2.70)$ & 0.024 & $2.30(1.32,4.08)$ & 0.004 \\
\hline
\end{tabular}

NLR: neutrophil-lymphocytes ratio, PLR: platelet-to-lymphocyte ratio, SIl: systemic immune-inflammation index, OR: odds ratio, Cl: confidence interval, Model 1: unadjusted, Model 2: adjusted for age, gender, BMI, smoking, alcohol assumption, educational status, marital status, regular exercise, self-report hypertension, triglyceride, high-density lipoprotein, low-density lipoprotein, fasting blood glucose and comorbidity 
Table 4 Association of immunity markers with risk of incident frailty in individuals without comorbidities

\begin{tabular}{|c|c|c|c|c|c|}
\hline Immunity markers & Event/N (\%) & Model 1 & & Model 2 & \\
\hline & & OR $(95 \% \mathrm{Cl})$ & $P$-value & $\mathrm{OR}(95 \% \mathrm{Cl})$ & $P$-value \\
\hline NLR & $142 / 956(14.85 \%)$ & & & & \\
\hline $\mathrm{Q} 1(\leq 1.26)$ & $24 / 232(10.34 \%)$ & Ref & Ref & Ref & Ref \\
\hline $\mathrm{Q} 2(>1.26, \leq 1.71)$ & $35 / 257(13.62 \%)$ & $1.36(0.79,2.39)$ & 0.275 & $1.20(0.63,2.33)$ & 0.580 \\
\hline Q3 $(>1.71, \leq 2.35)$ & $39 / 241$ (16.18\%) & $1.67(0.98,2.92)$ & 0.064 & $1.80(0.95,3.45)$ & 0.073 \\
\hline Q4 (> 2.35) & $44 / 226(19.47 \%)$ & $2.10(1.24,3.63)$ & 0.007 & $2.88(1.54,5.55)$ & 0.001 \\
\hline PLR & $142 / 956(14.85 \%)$ & & & & \\
\hline Q1 $(\leq 71.07)$ & $27 / 231$ (11.69\%) & Ref & Ref & Ref & Ref \\
\hline Q2 (> 71.07, $\leq 93.79)$ & $38 / 267$ (14.23\%) & $1.25(0.74,2.14)$ & 0.401 & $1.17(0.62,2.22)$ & 0.637 \\
\hline Q3 $(>93.79, \leq 126.81)$ & $36 / 236(15.25 \%)$ & $1.35(0.79,2.33)$ & 0.268 & $1.23(0.66,2.33)$ & 0.514 \\
\hline Q4 (> 126.81) & $41 / 222(18.47 \%)$ & $1.71(1.02,2.92)$ & 0.045 & $1.56(0.84,2.95)$ & 0.167 \\
\hline SII & $142 / 956(14.85 \%)$ & & & & \\
\hline Q1 ( $\leq 213.61)$ & $26 / 231(11.26 \%)$ & Ref & Ref & Ref & Ref \\
\hline Q2 $(>213.61, \leq 309.45)$ & $33 / 258(12.79 \%)$ & $1.15(0.67,2.00)$ & 0.614 & $1.32(0.67,2.65)$ & 0.420 \\
\hline Q3 (> 309.45, $\leq 445.76)$ & 39/247 (15.79\%) & $1.44(0.85,2.48)$ & 0.181 & $1.73(0.90,3.40)$ & 0.104 \\
\hline Q4 (> 445.76) & $44 / 220(20.00 \%)$ & $1.97(1.17,3.37)$ & 0.011 & $2.59(1.39,5.00)$ & 0.003 \\
\hline
\end{tabular}

NLR: neutrophil-lymphocytes ratio, PLR: platelet-to-lymphocyte ratio, SIl: systemic immune-inflammation index, OR: odds ratio, Cl: confidence interval, Model 1: unadjusted, Model 2: adjusted for age, gender, BMI, smoking, alcohol assumption, educational status, marital status, regular exercise, self-reported hypertension, triglyceride, high-density lipoprotein, low-density lipoprotein and fasting blood glucose

0.001) were associated with an increased risk of sarcopenia [19]. Similarly, Öztürk et al. included 419 individuals and reported a significant association of NLR with sarcopenia in a case-control study $(\mathrm{OR}=1.31$, 95\% CI: $1.06-$ 1.62, P-value $=0.013$ ) [20]. Liaw et al. also enrolled 3671 individuals aged $\geq 60$ years from the National Health and Nutrition Examination Survey (NHANES) III and found that elevated PLR levels were significantly associated with the risk of sarcopenia (OR $=1.001,95 \% \mathrm{CI}$ : $1.007-$ 1.009, P-value <0.001) [29]. In brief, previous studies showed that these immunity markers are associated with precursor syndromes and/or components of frailty, which is consistent with our findings that these immunity markers are associated with the risk of incident frailty in older adults in general.

\section{The possible mechanisms}

The mechanisms of the association of NLR and SII with incident frailty are not entirely known. Several pathways are involved in the association of the altered immune system with frailty. Neutrophils are important biomarkers of innate immunity, and platelets may contribute to immune function, whereas lymphocytes potentially reveal massive information on adaptive immunity [10]. NLR, PLR and SII are thought to reflect the balance between the innate and adaptive immune systems $[11,12]$. While, age-associated changes in the immune system could also lead to an increase in lowgrade, chronic systemic inflammation [30]. NLR, PLR and SII are proposed as inflammatory biomarkers.
Inflammation is widely recognized to be associated with frailty, such as for other inflammatory markers like Creactive protein, interleukin- 6 and tumor necrosis factor $\alpha$ [31-33]. The potential mechanism may be that inflammation is associated with reduced synthesis and activity of insulin-like growth factor I (IGF-I), which is essential for muscle regeneration and the maintenance of muscle integrity [34]. A loss of skeletal muscle strength and mass is a precursor syndrome and/or component of frailty.

\section{Strengths and limitations}

Several strengths exist in this study. First, we examined and validated the association of the NLR and SII levels with incident frailty in prospective, longitudinal and community-based cohorts. The generalization and reliability of our findings could be improved. Second, to validate our hypothesis, we conducted our study after adjusting for potential confounding factors that could affect these circulatory markers of immunity (such as BMI, physical activity and smoking) [35-37] and analyzed the association of these immunity markers with incident frailty in individuals with and without comorbidities. These sensitivity analyses attempted to rule out the potential effects of confounding factors. Third, these immunity markers (NLR and SII) obtained from complete blood counts are inexpensive, easy to measure and are reproducible in the laboratory. More importantly, due to the government of China providing free routine medical examinations for elderly individuals every year at community health service stations [38], 
these immunity markers could be developed annually by utilizing pre-existing annual routine medical examination data, not placing any extra burdens on the local government, community doctors or older adults.

The limitations were also presented in our study. Because of NLR, PLR and SII were measured at one point; subsequent temporal changes in these immunity markers were not captured. Therefore, longitudinal studies with repeated measurements during the follow-up may mitigate these limitations, improve the reliability, and illustrate the natural history of exposure-induced outcomes. In addition, we had not conducted any measurements on immune cell function or assessed systemic inflammation with markers such as CRP, interleukins 6 and interleukins 1 , and then much of this really does not relate to their biomarkers. Further studies would focus on exploring possible relationship between immune function and frailty in the future.

\section{Conclusion}

In conclusion, in this community-based study, we found that higher NLR and SII were associated with an increased risk of incident frailty in older adults in general. The clinical implications are that these immunity markers convey massive information on the future incidence of frailty and further reveal the importance of conducting interventions to improve the immune system to limit the incidence of frailty in older adults. Additionally, since NLR and SII levels are extensively used in the clinical field and are readily available assessments in the laboratory, they may be proposed as cost-effective predictors for the future incidence of frailty. More importantly, benefitting from free routine medical examinations in China, these immunity markers could be inspected annually without any additional medical burdens and should be used to conveniently predict the risk of frailty.

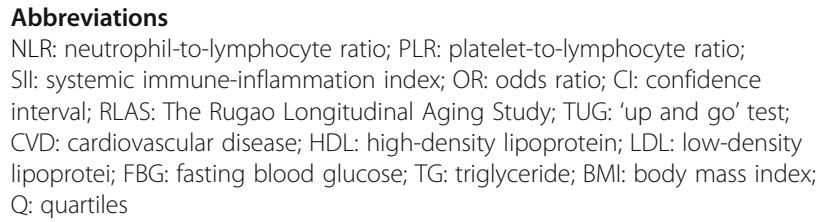

\section{Supplementary Information}

The online version contains supplementary material available at https://doi. org/10.1186/s12979-021-00257-6

Additional file 1: Supplemental Table S1. Characteristics of the study population stratified by quartiles of NLR levels at baseline. Supplemental Table S2. Characteristics of the study population stratified by quartiles of PLR levels at baseline. Supplemental Table S3. Characteristics of the study population stratified by quartiles of SII levels at baseline.

Supplemental Table S4. Association of immunity markers with risk of frailty at baseline. Supplemental Table S5. Association of immunity markers with risk of frailty among all individuals at baseline.

Supplemental Table S6. Association of immunity markers with risk of

frailty among individuals without comorbidity at baseline

\section{Acknowledgements}

We acknowledge all participants involved in the present study from the RuLAS. We acknowledge the support from the government of Rugao, the Public Health Bureau of Jiang'an Township and Rugao city, the Bureau of Civil Affairs, the Rugao People's Hospital and Human Phenome Institute, Fudan University, Shanghai, China.

\section{Authors' contributions}

Zhang H, Sun X, and Wang X: design research; Zhang H, Li Y, Jiang X, Liu, Z, Jin $L$ and Wang J: conducted the research; Zhang $H, H u Z$ and Hao $M$ : analyzed the data, performed the tactical analyses. Zhang $H$ and Wang $X$ : wrote the paper; Zhang $\mathrm{H}$ and Wang $\mathrm{X}$ : had primary responsibility for the final content; and all authors read and approved the final manuscript.

\section{Funding}

This work was supported by grants from the National Key R\&D Program of China (2018YFC2000400, 2018YFC2000400-3, 2018YFC2002000), National Natural Science Foundation of China-Youth Science Fund (3210040426) and Shanghai Municipal Science and Technology Major Project (2017SHZDZX01).

Availability of data and materials

The datasets generated and/or analyzed during the current study are available from the corresponding author on reasonable request.

\section{Declarations}

Ethics approval and consent to participate

This study was approved by the Human Ethics Committee of the School of Life Sciences of Fudan University. Informed consent was obtained from each participant.

Consent for publication

Not applicable.

\section{Competing interests}

The authors declare that they have no relevant financial interests.

\section{Author details}

${ }^{1}$ Human Phenome Institute, Fudan University, Shanghai, China. ${ }^{2}$ National Clinical Research Center for Aging and Medicine, Huashan Hospital, Fudan University, Shanghai, China. ${ }^{3}$ State Key Laboratory of Genetic Engineering, Collaborative Innovation Center for Genetics and Development, School of Life Sciences, Fudan University, Shanghai, China. ${ }^{4}$ Fudan Zhangjiang Institute, Shanghai, China. ${ }^{5}$ Key Laboratory of Arrhythmias, Ministry of Education, Department of Pathology and Pathophysiology, School of Medicine, Tongji University, Shanghai, China. ${ }^{6}$ Department of Big Data in Health Science, School of Public Health and the Second Affiliated Hospital, Zhejiang University School of Medicine, Hangzhou, China.

Received: 15 November 2021 Accepted: 30 November 2021 Published online: 03 January 2022

\section{References}

1. Clegg A, Young J, lliffe S, Rikkert MO, Rockwood K. Frailty in elderly people. Lancet (London, England). 2013;381(9868):752-62. https://doi.org/10.1016/ s0140-6736(12)62167-9.

2. O'Caoimh R, Sezgin D, O'Donovan MR, Molloy DW, Clegg A, Rockwood K, et al. Prevalence of frailty in 62 countries across the world: a systematic review and meta-analysis of population-level studies. Age Ageing. 2021; 50(1):96-104. https://doi.org/10.1093/ageing/afaa219.

3. Liu HX, Ding G, Yu WJ, Liu TF, Yan AY, Chen HY, et al. Association between frailty and incident risk of disability in community-dwelling elder people: evidence from a meta-analysis. Public Health. 2019;175:90-100. https://doi. org/10.1016/j.puhe.2019.06.010

4. Hanlon P, Nicholl BI, Jani BD, Lee D, McQueenie R, Mair FS. Frailty and prefrailty in middle-aged and older adults and its association with 
multimorbidity and mortality: a prospective analysis of 493737 UK biobank participants. Lancet Public Health. 2018;3(7):e323-e32. https://doi.org/10.101 6/s2468-2667(18)30091-4.

5. Aguayo GA, Vaillant MT, Donneau AF, Schritz A, Stranges S, Malisoux L, et al. Comparative analysis of the association between 35 frailty scores and cardiovascular events, cancer, and total mortality in an elderly general population in England: an observational study. PLoS Med. 2018;15(3): e1002543. https://doi.org/10.1371/journal.pmed.1002543.

6. Hoogendijk EO, Afilalo J, Ensrud KE, Kowal P, Onder G, Fried LP. Frailty: implications for clinical practice and public health. Lancet (London, England). 2019;394(10206):1365-75. https://doi.org/10.1016/s0140-6736(1 9)31786-6.

7. Wilson D, Jackson T, Sapey E, Lord JM. Frailty and sarcopenia: the potential role of an aged immune system. Ageing Res Rev. 2017;36:1-10. https://doi. org/10.1016/j.arr.2017.01.006.

8. Ferrucci L, Fabbri E. Inflammageing: chronic inflammation in ageing, cardiovascular disease, and frailty. Nat Rev Cardiol. 2018;15(9):505-22. https://doi.org/10.1038/s41569-018-0064-2.

9. Pansarasa O, Pistono C, Davin A, Bordoni M, Mimmi MC, Guaita A, et al. Altered immune system in frailty: genetics and diet may influence inflammation. Ageing Res Rev. 2019;54:100935. https://doi.org/10.1016/j.arr.2 019.100935.

10. Bonilla FA, Oettgen HC. Adaptive immunity. J Allergy Clin Immunol. 2010; 125(2 Suppl 2):S33-40. https://doi.org/10.1016/j.jaci.2009.09.017.

11. Hu B, Yang XR, Xu Y, Sun YF, Sun C, Guo W, et al. Systemic immuneinflammation index predicts prognosis of patients after curative resection for hepatocellular carcinoma. Clin Cancer Res Off J Am Assoc Cancer Res. 2014;20(23):6212-22. https://doi.org/10.1158/1078-0432.Ccr-14-0442.

12. Fest J, Ruiter R, Ikram MA, Voortman T, van Eijck CHJ, Stricker BH. Reference values for white blood-cell-based inflammatory markers in the Rotterdam study: a population-based prospective cohort study. Sci Rep. 2018;8(1): 10566. https://doi.org/10.1038/s41598-018-28646-w.

13. van der Willik KD, Fani L, Rizopoulos D, Licher S, Fest J, Schagen SB, et al. Balance between innate versus adaptive immune system and the risk of dementia: a population-based cohort study. J Neuroinflammation. 2019; 16(1):68. https://doi.org/10.1186/s12974-019-1454-z

14. Fani L, van der Willik KD, Bos D, Leening MJG, Koudstaal PJ, Rizopoulos D, et al. The association of innate and adaptive immunity, subclinical atherosclerosis, and cardiovascular disease in the Rotterdam study: a prospective cohort study. PLoS Med. 2020;17(5):e1003115. https://doi.org/1 0.1371/journal.pmed.1003115.

15. Fest J, Ruiter TR, Groot Koerkamp B, Rizopoulos D, Ikram MA, van Eijck CHJ, et al. The neutrophil-to-lymphocyte ratio is associated with mortality in the general population: the Rotterdam study. Eur J Epidemiol. 2019;34(5):46370. https://doi.org/10.1007/s10654-018-0472-y.

16. Mathur K, Kurbanova N, Qayyum R. Platelet-lymphocyte ratio (PLR) and allcause mortality in general population: insights from national health and nutrition education survey. Platelets. 2019;30(8):1036-41. https://doi.org/10.1 080/09537104.2019.1571188.

17. Hou P, Xue HP, Mao XE, Li YN, Wu LF, Liu YB. Inflammation markers are associated with frailty in elderly patients with coronary heart disease. Aging. 2018;10(10):2636-45. https://doi.org/10.18632/aging.101575

18. Guo J, Zhang H, Li Y, Hao M, Shi G, Wang J, et al. Neutrophil-lymphocyte ratio as a predictor of slow gait speed in older adults: the Rugao longitudinal aging study. Exp Gerontol. 2021;152:111439. https://doi.org/10.1 016/j.exger.2021.111439.

19. Zhao WY, Zhang Y, Hou LS, Xia X, Ge ML, Liu XL, et al. The association between systemic inflammatory markers and sarcopenia: results from the West China health and aging trend study (WCHAT). Arch Gerontol Geriatr. 2021;92:104262. https://doi.org/10.1016/j.archger.2020.104262.

20. Öztürk ZA, Kul S, Türkbeyler IH, Sayıner ZA, Abiyev A. Is increased neutrophil lymphocyte ratio remarking the inflammation in sarcopenia? Exp Gerontol. 2018;110:223-9. https://doi.org/10.1016/j.exger.2018.06.013.

21. Liu Z, Wang Y, Zhang Y, Chu X, Wang Z, Qian D, et al. Cohort profile: the Rugao longevity and ageing study (RuLAS). Int J Epidemiol. 2016;45(4) 1064-73. https://doi.org/10.1093/ije/dyv101.

22. Fried LP, Tangen CM, Walston J, Newman AB, Hirsch C, Gottdiener J, et al. Frailty in older adults: evidence for a phenotype. J Gerontol A Biol Sci Med Sci. 2001;56(3):M146-56. https://doi.org/10.1093/gerona/56.3.m146.

23. Sun XH, Ma T, Yao S, Chen ZK, Xu WD, Jiang XY, et al. Associations of sleep quality and sleep duration with frailty and pre-frailty in an elderly population Rugao longevity and ageing study. BMC Geriatr. 2020;20(1):9 https://doi.org/10.1186/s12877-019-1407-5.

24. Collerton J, Martin-Ruiz C, Davies K, Hilkens CM, Isaacs J, Kolenda C, et al. Frailty and the role of inflammation, immunosenescence and cellular ageing in the very old: cross-sectional findings from the Newcastle $85+$ study. Mech Ageing Dev. 2012;133(6):456-66. https://doi.org/10.1016/j.mad.2 012.05.005.

25. Peel NM, Kuys SS, Klein K. Gait speed as a measure in geriatric assessment in clinical settings: a systematic review. J Gerontol A Biol Sci Med Sci. 2013; 68(1):39-46. https://doi.org/10.1093/gerona/gls174.

26. Woo J. Walking speed: a summary Indicator of frailty? J Am Med Dir Assoc. 2015;16(8):635-7. https://doi.org/10.1016/j.jamda.2015.04.003.

27. Middleton A, Fritz SL, Lusardi M. Walking speed: the functional vital sign. J Aging Phys Act. 2015;23(2):314-22. https://doi.org/10.1123/japa.2013-0236.

28. Borges TC, Gomes TL, Pichard C, Laviano A, Pimentel GD. High neutrophil to lymphocytes ratio is associated with sarcopenia risk in hospitalized cancer patients. Clin Nutr (Edinburgh, Scotland). 2021;40(1):202-6. https://doi.org/1 0.1016/j.clnu.2020.05.005

29. Liaw FY, Huang CF, Chen WL, Wu LW, Peng TC, Chang YW, et al. Higher platelet-to-lymphocyte ratio increased the risk of sarcopenia in the community-dwelling older adults. Sci Rep. 2017;7(1):16609. https://doi.org/1 0.1038/s41598-017-16924-y.

30. Franceschi C, Bonafè M, Valensin S, Olivieri F, De Luca M, Ottaviani E, et al. Inflamm-aging. An evolutionary perspective on immunosenescence. Ann N Y Acad Sci. 2000;908(1):244-54. https://doi.org/10.1111/j.1749-6632.2000. tb06651.x.

31. Marcos-Pérez D, Sánchez-Flores M, Proietti S, Bonassi S, Costa S, Teixeira JP, et al. Association of inflammatory mediators with frailty status in older adults: results from a systematic review and meta-analysis. GeroScience. 2020;42(6):1451-73. https://doi.org/10.1007/s11357-020-00247-4.

32. Langmann GA, Perera S, Ferchak MA, Nace DA, Resnick NM, Greenspan SL. Inflammatory markers and frailty in long-term care residents. J Am Geriatr Soc. 2017:65(8):1777-83. https://doi.org/10.1111/jgs.14876.

33. Walker KA, Walston J, Gottesman RF, Kucharska-Newton A, Palta P, Windham BG. Midlife systemic inflammation is associated with frailty in later life: the ARIC study. J Gerontol A Biol Sci Med Sci. 2019;74(3):343-9. https:// doi.org/10.1093/gerona/gly045.

34. Sartori R, Romanello V, Sandri M. Mechanisms of muscle atrophy and hypertrophy: implications in health and disease. Nat Commun. 2021;12(1): 330. https://doi.org/10.1038/s41467-020-20123-1.

35. Furuncuoğlu Y, Tulgar S, Dogan AN, Cakar S, Tulgar YK, Cakiroglu B. How obesity affects the neutrophil/lymphocyte and platelet/lymphocyte ratio, systemic immune-inflammatory index and platelet indices: a retrospective study. Eur Rev Med Pharmacol Sci. 2016;20(7):1300-6.

36. Bauer M, Fink B, Thürmann L, Eszlinger M, Herberth G, Lehmann I. Tobacco smoking differently influences cell types of the innate and adaptive immune system-indications from CpG site methylation. Clin Epigenetics 2015;7(1):83. https://doi.org/10.1186/s13148-016-0249-7.

37. Duggal NA, Niemiro G, Harridge SDR, Simpson RJ, Lord JM. Can physical activity ameliorate immunosenescence and thereby reduce age-related multi-morbidity. Nat Rev Immunol. 2019;19(9):563-72. https://doi.org/10.103 8/s41577-019-0177-9.

38. China NHCotPsRo. The national basic public health service specification (third edition) (in Chinese), vol. 3: Gazette of the National Health and Family Planning Commission of People's Republic of China; 2017. p. p53-6.

\section{Publisher's Note}

Springer Nature remains neutral with regard to jurisdictional claims in published maps and institutional affiliations. 